\title{
Idiopathic pulmonary fibrosis and sleep disorders: no longer strangers in the night
}

\author{
Sophia Schiza ${ }^{1,9}$, Charalampos Mermigkis ${ }^{1,9}$, George A. Margaritopoulos ${ }^{2,3}$, \\ Zoi Daniil ${ }^{4}$, Sergio Harari ${ }^{5}$, Venerino Poletti ${ }^{6}$, Elizabetta A. Renzoni ${ }^{7}$, \\ Olga Torre ${ }^{5}$, Dina Visca ${ }^{7}$, Isolde Bouloukaki ${ }^{1}$, George Sourvinos ${ }^{8}$ and \\ Katerina M. Antoniou ${ }^{2}$
}

Affiliations: ${ }^{1}$ Dept of Thoracic Medicine, Sleep Disorders Center, Medical School, University of Crete, Heraklion, Greece. ${ }^{2}$ Dept of Thoracic Medicine, Interstitial Lung Disease Unit \& Laboratory of Molecular and Cellular Pneumonology, Medical School, University of Crete, Heraklion, Greece. ${ }^{3}$ Dept of Respiratory Medicine, General Hospital of Kavala, Kavala, Greece. ${ }^{4}$ Respiratory Medicine Dept, University Hospital of Larissa, Larissa, Greece. ${ }^{5}$ U.O. di Pneumologia e Terapia Semi-Intensiva Respiratoria, Servizio di Fisiopatologia Respiratoria ed Emodinamica Polmonare, Ospedale San Giuseppe - MultiMedica, Milan, Italy. ${ }^{6}$ Dept of Diseases of the Thorax/Pulmonology Unit, Ospedale GB Morgagni, Forli, Italy. ${ }^{7}$ Interstitial Lung Disease Unit, Royal Brompton Hospital, London, UK. ${ }^{8}$ Laboratory of Virology, Medical School, University of Crete, Heraklion, Greece. ${ }^{9}$ Both authors contributed equally.

Correspondence: Katerina M. Antoniou, Dept of Thoracic Medicine, Medical School, University of Crete, Heraklion, 71110 Crete, Greece. E-mail: kantonioudmed.uoc.gr

ABSTRACT The prevalence of obstructive sleep apnoea (OSA) is continuously increasing in patients with idiopathic pulmonary fibrosis (IPF) and, for the first time, the recent IPF guidelines recognise OSA as an important associated comorbidity that can affect patient's survival. Thus, it becomes conceivable that clinicians should refer patients with newly diagnosed IPF to sleep centres for the diagnosis and treatment of OSA as well as for addressing issues regarding the reduced compliance of patients with continuous positive airway pressure therapy. The discovery of biomarkers common to both disorders may help early diagnosis, institution of the most appropriate treatment and follow-up of patients. Better understanding of epigenetic changes may provide useful information about pathogenesis and, possibly, development of new drugs for a dismal disease like IPF.

@ERSpublications

It is now believed that IPF and sleep disorders can coexist in the same patient http://ow.ly/LXPSL

\section{Introduction}

Based on recent literature reporting an increased incidence of obstructive sleep apnoea (OSA) in patients with idiopathic pulmonary fibrosis (IPF), for the first time, the most recent IPF guidelines include OSA among IPF-associated comorbidities [1]. The paucity of studies related to continuous positive airway pressure (CPAP) treatment in this patient group and the need to intensify research in this area were highlighted by a European Respiratory Society (ERS)-endorsed meeting entitled "IPF and sleep: common pathways and implications in therapy" (December, 2013; Athens, Greece). IPF is a fatal disease with median survival of 3 years [2]. Identification and treatment of complicating comorbidities such as OSA and pulmonary hypertension $(\mathrm{PH})$ may have a significant impact on survival in this devastating disease. In this review, we aim to highlight the progress achieved so far and underline challenges for future studies in this field.

Received: Sept 272014 | Accepted after revision: Nov 092014

Conflict of interest: None declared.

Provenance: Submitted article, peer reviewed.

Copyright OERS 2015. ERR articles are open access and distributed under the terms of the Creative Commons Attribution Non-Commercial Licence 4.0. 


\section{Sleep in IPF}

In healthy individuals, sleep represents a state of restoration covering approximately one-third of human life. The deleterious effects of disease processes are present both during wakefulness and sleep, although sleep is often overlooked by physicians. Sleep hypoventilation, especially during the vulnerable rapid eye movement (REM) period, well tolerated by healthy individuals, may represent a potentially hazardous occurrence in patients with chronic respiratory diseases $[3,4]$. During REM sleep, the diaphragm remains the only active respiratory muscle, while accessory muscles are temporarily not functional (normal REM-related muscle paralysis).

So far, research has mainly focused on patients with chronic obstructive pulmonary disease (COPD). Significant changes in oxygenation parameters and/or coexisting sleep disordered breathing (sometimes called "overlap syndrome") have been described in patients with severe COPD, particularly during the vulnerable REM sleep period $[5,6]$. Less research has been performed in patients with restrictive lung diseases such as IPF. Although the positive findings relating to pirfenidone and nintedanib $[7,8]$ have changed the outlook for patients with IPF, both drugs have been found to reduce rather than halt disease progression, and neither has been shown to significantly improve quality of life. Sleep disturbances are likely to play a major role in overall quality of life as well as in morbidity and mortality in this patient group (table 1) [9-11].

Published studies related to sleep in IPF should be divided into those performed before and after publication of the 2002 American Thoracic Society (ATS)/ERS criteria for the diagnosis for IPF [12]. The limited number of studies from the mid-1980s to the 1990s included small numbers of patients with a variety of restrictive lung diseases. By contrast, studies performed after the 2002 guidelines were published mostly included only patients with a confirmed diagnosis of IPF. This is important, as IPF exclusively affects the lungs, while other interstitial lung diseases (ILDs) can occur on a background of systemic disease, such as connective tissue disease or sarcoidosis, affecting multiple organs/systems that may themselves influence sleep quality and/or sleep-related disorders in a number of ways. Recent methodological differences, including a change in the definition of hypopnoeas as well as newer and more sensitive equipment used for event detection, also make the comparison between older and more recent studies difficult [13].

Overall, sleep is found to be markedly disturbed across studies in patients with IPF. Most studies agree on the findings of abnormal sleep macro- and micro-architecture, respiratory breathing patterns, and oxygen desaturation with features of hypoventilation, especially during the vulnerable REM period. However, OSA was reported as uncommon in the older studies, while it has instead been described as quite frequent in the more recent reports.

BYE et al. [14], in a study from the mid-1980s that investigated patients with ILD (13 ILD patients, three with IPF) using standard polysomnography (PSG), showed that patients with ILD had sleep breathing frequency persistently above the normal range: seven out of nine who did not snore had marked desaturations during REM sleep, and the other two had desaturations in both REM and non-REM sleep.

TABLE 1 Sleep in idiopathic pulmonary fibrosis

Sleep macro- and micro-architecture

Increased stage 1 sleep

Decreased REM and slow-wave sleep

Decreased sleep efficiency

Increased WASO

Increased arousal index

Respiratory pattern

Nocturnal oxygenation parameters

Sleep disordered breathing

Other sleep problems
Increased respiratory frequency during sleep

Rapid and shallow breathing (especially during REM sleep)

Episodic desaturation during REM sleep

Desaturation during NREM sleep

Desaturation due to respiratory events (apnoeas and hypopnoeas)

Increased incidence of obstructive sleep apnoea

Increased periodic leg movements during sleep

Insomnia

Nocturnal cough

REM: rapid eye movement; WASO: wake time after sleep onset; NREM: non-REM. 
As stated by those authors, OSA was an unexpected feature in two out of four patients who snored. Perez-Padilla et al. [15], in a study from the same time period, observed that patients with ILD (11 ILD patients, seven patients with IPF) had poor sleep quality with less time in REM sleep, increased stage 1 sleep and marked sleep fragmentation compared with age- and sex-matched controls. The apnoeahypopnoea index (AHI), defined as the number of apnoea and hypopnoea events per hour of sleep, was found to be low even though hypoxaemia and obesity were noted in several of these patients. ILD patients had a higher respiratory frequency than controls, during both wakefulness and sleep. Respiratory frequency during sleep showed no significant change in IPF patients in contrast to the noted decrease in the control group. The authors hypothesised that the lack of change might be due to the maintenance of reflexes causing rapid, shallow breathing during sleep. The same breathing pattern was observed in a more recent study by MermigKis et al. [16] including 15 IPF patients with diagnosis based on the 2002 ATS/ERS criteria and 15 matched control subjects. In contrast, McNicholas et al. [17], in an older study including seven patients (five with IPF, two with farmer's lung), demonstrated that respiratory frequency declined significantly between wakefulness and sleep in ILD patients. However, the authors do acknowledge a possible confounding effect in their own study due to anxiety in the period preceding the study when the respiratory frequency was measured for the period of wakefulness.

OSA is a condition characterised by cessation of regular breathing during sleep. Apnoea is a complete cessation of respiration and hypopnoea is partial or reduced respiration. For the diagnosis of sleep apnoea, each apnoeic or hypopnoeic event must last a minimum of $10 \mathrm{~s}$. Each respiratory event generally results in repeated arousals from sleep as well as nocturnal hypoxaemia. OSA is considered mild if the AHI is $\geqslant 5$ events per hour but $<15$ events per hour, moderate if $\geqslant 15$ events per hour but $<30$ events per hour, and severe if $\geqslant 30$ events per hour.

The first study reporting the need for a high awareness for OSA in IPF population was a retrospective study by Mermigkis et al. [18] in 18 patients with IPF who had been referred for overnight PSG to investigate complaints suggestive of sleep disordered breathing. OSA was confirmed in 11 patients and the rest were diagnosed with upper airway resistance syndrome or primary snoring. The authors pointed out that despite growing awareness of OSA among healthcare providers, treating physicians may defer sleep testing because IPF is characterised by such a rapidly progressive course, leading them to focus on more acute problems such as dyspnoea and limitations in daily activities. This may be the case as among 875 patients with IPF admitted to the Cleveland Clinic (Cleveland, OH, USA) from 2001 to 2005, only 18 were referred to the sleep centre. Obesity and the magnitude of the pulmonary function test impairment were recognised as predictor factors for OSA. Restrictive pulmonary diseases are characterised by decreased lung volumes that can reduce the upper airway stability and increase resistance due to a decreased traction on the upper airway. These changes can facilitate upper airway collapse, especially during REM sleep when functional residual capacity is further reduced due to the inactivity of the intercostal muscles [19-22]. However, this is not enough to draw the conclusion that OSA is a direct consequence of IPF as multiple factors are possibly involved in the pathogenesis of OSA in IPF, an area that demands intense future research. Another significant finding of this study was the increased frequency of restless-leg syndrome and periodic leg movements during sleep in this population. The first prospective study on the topic OSA in IPF was performed in the USA by LANCASTER et al. [23], including 50 IPF patients. OSA was diagnosed in $88 \%$ of subjects. 10 (20\%) subjects had mild OSA and 34 (68\%) had moderate-to-severe OSA. Neither the Epworth Sleepiness Scale nor the Sleep Apnoea Scale of Sleep Disorders Questionnaire, alone or in combination, was a strong screening tool, making an evaluation by a sleep physician necessary. The study did not demonstrate an inverse relationship between forced vital capacity (FVC) and AHI, contrary to the previously described theory that decreased lung volumes predispose to OSA by reducing the upper airway stability and increasing resistance due to a decreased traction on the upper airway. The authors claimed that the lack of inverse correlation between the pulmonary function testing (PFT) data and the severity of sleep apnoea may be explained by the use of PFTs performed with the patient in the upright or standing position. FVCs obtained with the patient in the supine position may be more accurate at predicting sleep lung volumes, and may better display interdependence between the upper airway and lung volumes during sleep. A limitation of the study was the fact that the included patients were receiving corticosteroids or other treatments for IPF. This was excluded in a multicentre study from Greece by Mermigkis et al. [24] in which PSG was performed in 34 treatment-naïve patients soon after the diagnosis of IPF. 15 (44\%) subjects had mild and five (15\%) had moderate-to-severe OSA, while 14 (41\%) subjects were normal. The last study showing again that OSA is a common comorbidity in IPF was a study from Turkey by PIHTILI et al. [25]. Based on these studies, the incidence of OSA is high in IPF patients, even though it should be mentioned that IPF is known to occur mostly in those over the age of 60 years, an age where OSA increases even among normal people. Data from the Sleep Heart Health Study (SHHS) show, in the 60-69-year age group, prevalences of mild and moderate-to-severe OSA of 32\% and 19\%, respectively [26]. Based on these data, 51\% of people in the 60-69-year age group had an AHI above 5 events per hour, the 
upper normal value based on current criteria. In comparison, the studies by LANCASTER et al. [23] and Mermigkis et al. [24] reported an AHI $>5$ events per hour in $88 \%$ and $59 \%$ of the included IPF patients, respectively. These percentages are higher than those seen in this age group in the SHHS. The main difference between the studies in IPF patients is the fact that LANCASTER et al. [23] found the majority of patients (68\%) had moderate-to-severe OSA (AHI >15 events per hour), while MERMIGKIs et al. [24] reported mostly (44\%) mild OSA (AHI 5-15 events per hour). These differences may be related to the fact that Mermigkis et al. [24] included only treatment-naïve patients while LANCASTER et al. [23] included patients treated with corticosteroids, which may increase OSA as a result of fat deposition in the neck area. In addition, the body mass index (BMI) of included patients in the study by MermigKIs et al. [24] was significantly lower than the BMI of patients in the study by LANCASTER et al. [23].

The role of underlying OSA in the general health status of IPF patients is probably more important than has been recognised. There are a few published studies $[27,28]$ reporting that some features related to OSA, such as increased gastro-oesophageal reflux, exaggerated intrathoracic mechanical strains or intermittent hypoxia with oxidative stress, could somehow favour the development of IPF or impact the progression of the disease. In addition, it is well known that OSA has been increasingly linked to cardiovascular and cerebrovascular morbidity and mortality, and this association makes OSA a serious, potentially life-threading condition $[29,30]$. IPF patients with OSA are obviously not an exception to such issues. It is not a surprise that the most recent IPF guidelines [1] include OSA among the IPF-associated comorbidities. Furthermore, they recognise the paucity of studies related to CPAP treatment in this patient group and reinforce the need for intense research in this field. Mermigkis et al. [31] recognised that CPAP initiation in this population may be problematic for a variety of reasons, with high incidence of CPAP unacceptance or poor compliance, factors that can only be eliminated through intense follow-up by a well-organised sleep centre. The authors claimed that it would be easy to give up after a CPAP titration has been unsuccessful for multiple reasons, such as cough, claustrophobia or insomnia, and to characterise these patients as ineligible to receive CPAP therapy. However, the next step is simply to accept the well-known course of the disease that leads to disability and, finally, death. There are always solutions such as heated humidification even from the first CPAP initiation during the titration study, which may improve one of the most disabling symptoms, namely dry nocturnal cough. In addition, one crucial issue is to recognise sleep disorders in IPF patients in the early stages of the disease and not when they are close to the death when the possibility of CPAP unacceptance dramatically rises. The first study in this field [32] included 12 IPF patients with moderate-to-severe OSA started on CPAP therapy. Effective CPAP treatment in IPF patients with OSA resulted in a significant improvement in daily living activities based on the Functional Outcome of Sleep Questionnaire (FOSQ), a specific follow-up instrument that assesses the daily effects of poor sleep caused by sleep disorders. A statistically significant improvement was observed in the FOSQ at 1,3 and 6 months after CPAP initiation. The authors recognised the need for further studies to assess not only the negative influence of untreated OSA on the quality of life of IPF patients, but also the possible contribution of OSA to IPF progression and mortality. Another study by KolILEKAs et al. [33] in a cohort of 31 treatment-naïve, newly diagnosed, consecutive IPF patients reported that intermittent sleep oxygen desaturation significantly exceeds that of maximal exercise and is associated with survival in IPF patients. Among the included subjects, 12 (38.7\%) patients had mild and 16 (51.6\%) patients had moderate-to-severe OSA. The main limitation of this study was that only four patients were treated with CPAP. Even though it was interesting and they were all alive at the reporting of the study, one could speculate that CPAP might have offered a survival benefit. In a very recent multicentre study by Mermigkis et al. [34], CPAP therapy was initiated in 92 treatment-naïve, newly diagnosed, consecutive IPF patients with moderate-to-severe OSA. Patients were divided into a poor and a good CPAP compliance group. All subjects completed multiple quality-of-life and sleep instruments (FOSQ, Pittsburgh Sleep Quality Index, Epworth Sleepiness Scale, Fatigue Severity Scale, Short Form 36 Health Survey and Beck Depression Inventory) before CPAP initiation and 1 year after the start of CPAP treatment. The good CPAP compliance group showed statistically significant improvement in all used quality-of-life and sleep instruments after 1 year's CPAP treatment. However, the poor CPAP compliance group showed significant, smaller changes only in a minority of the used instruments. Interestingly, during the 24-month follow-up period after CPAP initiation, three patients from the poor CPAP compliance group died, whereas all patients from the good CPAP compliance group remained alive. This is the first study to report that effective CPAP treatment in IPF patients with comorbid moderate-to-severe OSA results in a significant improvement in daily living activities, and quality of sleep and life. In addition, it provides the first evidence that treatment of comorbidities such as OSA may also influence mortality in IPF, a fatal disease (IPF is not a cancer but acts like a cancer) with no effective treatment so far.

In IPF, sleep is probably not a restorative state but a marathon made up of 100-m races every time patients go into REM sleep. Obviously, PSG is expensive and cannot be advised in all IPF patients. In addition, in this population, OSA cannot be accurately predicted by questionnaires [23] or based on symptoms that 
most non-sleep physicians look for, such as excessive daytime sleepiness [18, 19]. The main complaint in IPF patients with OSA is daytime fatigue, a complaint with a multifactorial profile that is difficult to assess $[11,24]$. In addition, based on the recent PSG studies in this population, the majority of the respiratory events are hypopnoeas and the accurate recognition of these needs special equipment and follows new terminology [13, 23-25].

\section{Sleep apnoea and PH}

OSA is associated with repetitive nocturnal arterial oxygen desaturation and hypercapnia. In animal models, intermittent hypoxia has been shown to cause $\mathrm{PH}$, pulmonary arteriolar remodelling and right ventricular hypertrophy [35-37]. Exposure to cyclic intermittent hypoxia decreases endothelial nitric oxide synthase (eNOS) expression and nitric oxide level, and increases endothelin-1 expression in pulmonary arteries in rats, thus predisposing the vasculature to increased tone, altered remodelling, proliferation and endothelial injury [38].

$\mathrm{PH}$ is relatively common in patients with OSA and is thought to be associated with pulmonary vascular remodelling. The prevalence of $\mathrm{PH}$ in OSA without clinically identifiable cardiopulmonary disease has been found to be $20-40 \%$ [39].

A retrospective analysis of 83 patients with OSA who underwent right heart catheterisation showed $\mathrm{PH}$ in 58 (70\%) patients [40]. Among these, 18 patients had pulmonary arterial hypertension (PAH). No significant differences were observed between the $\mathrm{PH}$ and non-PH groups regarding AHI. The $\mathrm{PH}$ group experienced significantly more frequent nocturnal desaturation than the non- $\mathrm{PH}$ group; patients with $\mathrm{PAH}$ had the longest duration of nocturnal desaturation. Most patients had mild or moderate elevations in pulmonary arterial pressure (PAP); however, a total of 27 patients had severe PH (mean PAP (mPAP) $\geqslant 40 \mathrm{mmHg}$ ). Patients with severe $\mathrm{PH}$ had more nocturnal desaturation, worse haemodynamics and greater mortality than patients with mild or moderate $\mathrm{PH}$ or no PH. Right ventricular systolic pressure, BMI, female sex and duration of nocturnal desaturation were the strongest correlates of $\mathrm{PH}$. Given the retrospective nature of the study, a selection bias can cause the prevalence estimate of $\mathrm{PH}$ in association with OSA to be high. In addition, several comorbidities that might have an effect on the presence of $\mathrm{PH}$ could not be properly excluded.

A 3-6-month trial of nocturnal CPAP treatment has been shown to decrease PAP, as derived by Doppler echocardiography $[41,42]$.

A recent prospective study on patients with a diagnosis of $\mathrm{PH}$ confirmed by right heart catheterisation analysed the prevalence and characteristics of sleep disorders by polygraphy. Among 169 patients with $\mathrm{PH}$, 45 (26.6\%) PH patients were found to suffer from sleep disorders. Of these, 27 (16\%) patients had OSA and $18(10.6 \%)$ patients had central sleep apnoea (CSA). The severity of sleep apnoea was mild to moderate, with a mean AHI of 20 events per hour. OSA mainly occurred in patients with chronic thromboembolic $\mathrm{PH}(\mathrm{CTEPH})$ and COPD-associated PH; most patients with CSA were seen in idiopathic $\mathrm{PAH}, \mathrm{CTEPH}$ and "other" diagnoses of PH [43].

\section{IPF and PH}

The prevalence of PH complicating the course of patients with IPF has been reported as occurring in $32-85 \%$ of patients. These widely varying estimates reflect differing patient populations and disease severity among different studies. In one study, $36 \%$ of patients assessed at the time of transplantation evaluation had $\mathrm{PH}$ but $85 \%$ of the same patient cohort had $\mathrm{PH}$ at the time of transplantation [44]. The presence of $\mathrm{PH}$ in IPF is associated with higher mortality and its development contributes to the deterioration of IPF patients [45,46]. Moreover, in severe diffuse lung disease, pulmonary vascular resistance has been shown to strongly predict death within 1 year independent of disease severity or diagnosis of IPF [47]. Noninvasive diagnostic tests such as echocardiography, 6-min walking distance, distance-saturation product and oxygen saturation measured by pulse oximetry $\left(\mathrm{SpO}_{2}\right)$ seem to perform poorly in detecting PAH in IPF patients [48]. However, PH is mild in most IPF patients. In one series, only about $10 \%$ of patients with IPF listed for transplantation had severe $\mathrm{PH}$, as defined by a mPAP $>40 \mathrm{mmHg}$. To date, there is not sufficient evidence that the drugs currently used for PAH are safe and effective in patients with $\mathrm{PH}$ associated with IPF [49]. Thus, the use of drugs currently approved for PAH in patients with IPF is not recommended until further data are available; lung transplantation is still the best option for these patients.

\section{Nocturnal hypoxia and supplemental oxygen in IPF}

As mentioned in the previous sections, nocturnal oxygen desaturation is frequent in patients with IPF and, more generally, in patients with ILD [50-52]. CoRTE et al. [51] found that 36\% of 139 patients with ILD had arterial oxygen saturation $\left(\mathrm{SaO}_{2}\right)<90 \%$ for $>10 \%$ of their sleep. An elevated desaturation index 
(number of desaturation events of $>4 \%$ per hour of sleep) was a significant independent predictor of worse survival and significantly correlated with $\mathrm{PH}$ in patients with limited (diffusing capacity of the lung for carbon monoxide $>35 \%$ ) fibrosis. Interestingly, only a loose correlation was found between nocturnal hypoxaemia and daytime hypoxia (at rest or during exercise) [15, 51]. Furthermore, several studies have reported that nocturnal desaturation in ILD is only loosely correlated, if at all, with the severity of lung function impairment $[50,51]$. The possible link with $\mathrm{PH}$ was also reported by KoliLEKAs et al. [33] in a recent prospective study of 31 patients with IPF, where the lowest sleep $\mathrm{SpO}_{2}$ was linked to survival and estimated right ventricle systolic pressure (RVSP). In another recent prospective study of 33 patients with IPF, RVSP correlated with mean $\mathrm{SaO}_{2}$ during sleep, but not with FVC. Again, no significant correlation was observed between nocturnal saturation and day time arterial oxygen tension [53].

Although overnight desaturation events in patients with ILD can be a consequence of OSA, it is clear that they can also occur in the absence of sleep disordered breathing. As stated in the previous sections, there is a wide variability in the reported prevalence of OSA in ILD patients, from $17 \%$ to $88 \%$, probably depending on a range of differences in the populations studied, including different referral patterns, prevalence of obesity, ethnicity and corticosteroid treatment [18, 23, 25, 54, 55]. However, a significant proportion of patients with IPF have overnight oxygen desaturation independently of the presence of OSA. Mermigkis et al. [31] reported sleep oxygenation abnormalities in a group of 34 IPF patients and found evidence of mild OSA syndrome in 15 and severe OSA syndrome in five patients. However, even in the 14 IPF patients with no evidence of OSA, a mean \pm SD of $12.4 \pm 22.7 \%$ of total sleep time was spent with a $\mathrm{SaO}_{2}$ $<90 \%$, with an average oxygen desaturation nadir of $85 \%$, not significantly different from patients with evidence of OSA. In the study by PITSIOU et al. [53], all patients were reported to have significant nocturnal desaturation: 23 out of the 33 prospectively investigated IPF patients had a normal AHI $<5$ events per hour, with eight having an AHI (between 5 and 15 events per hour) and only two patients with an AHI >15 events per hour. Further, larger studies in different patient populations, allowing stratification for potential covariates such as ethnicity and BMI, are required to study the prevalence of the different patterns of overnight desaturation in IPF and the mechanisms involved.

The most compelling reason to treat nocturnal hypoxia would be the prevention of $\mathrm{PH}$. The development of $\mathrm{PH}$ in ILD is a strong predictor of poor survival $[46,56,57]$. Unfortunately, the use of anti- $\mathrm{PH}$ treatments in this group of patients has not generated much optimism to date, as outlined in the previous sections and by a recent placebo-controlled trial of bosentan in patients with idiopathic interstitial pneumonias and $\mathrm{PH}$ [58]. The mechanisms thought to contribute to PH in ILD vary, and include hypoxic vasoconstriction, vascular remodelling, vessel ablation, vascular occlusion and compression. Nocturnal desaturation could play a significant role in PH development, although very few data are available in ILD patients. In COPD, episodes of sleep-related oxygen desaturation are frequent and more severe than daytime hypoxia. In the majority of cases, these are not due to OSA but to alveolar hypoventilation and/or ventilation-perfusion mismatching [59,60], and are often accompanied by rises in PAP of as much as 15-20 mmHg from its baseline value, at a level similar to that observed during exercise [61-65]. In COPD, long-term oxygen treatment (LTOT) for $\geqslant 18 \mathrm{~h}$ a day has been linked to improved survival and stabilisation or reduction in rate of progression of $\mathrm{PH}$ [66-69].

Correction of nocturnal hypoxia with supplemental oxygen would therefore seem to be a simple adjunct to treatment in patients with ILD. However, there is a dearth of studies addressing this question in patients with IPF or with ILD at large. The only randomised controlled trial of LTOT in ILD was aimed at patients with resting hypoxia (37 patients randomised to receive oxygen and 25 no oxygen) and, therefore, with advanced-stage fibrotic ILD, and is unpublished (A. Braghiroli, S. Maugeri Foundation, IRCCS, Veruno, Italy; personal communication) [70]. No difference in survival at 1, 2 and 3 years was observed between the two groups, although the absence of further data makes this finding difficult to interpret. Furthermore, no study has investigated the effects of treating nocturnal hypoxia in patients with less advanced disease, in whom there may be more scope to prevent irreversible changes to the pulmonary vasculature. Similarly, data are needed as guidance on which threshold of desaturation and duration of desaturation events should be used to trigger use of supplemental oxygen.

A potential benefit of recognising and correcting nocturnal hypoxaemia with supplemental oxygen would be the improvement of daytime fatigue, with the potential to significantly improve quality of life in these patients. Fatigue is one of the most frequently reported symptoms in ILD and has a marked impact on patients' wellbeing [71]. MermigKis et al. [16] reported a significant correlation between mean nocturnal $\mathrm{SaO}_{2}$ and fatigue scores in patients with IPF and found that nocturnal hypoxia explained up to $45 \%$ of the variance on fatigue scores. Although, as reported in the previous section, a benefit in sleep-related quality of life was reported following institution of CPAP for patients with IPF and OSA, studies are needed to assess the hypothesis that the use of supplemental oxygen could beneficially affect fatigue and quality of life in patients with ILD. 
In summary, the effects of nocturnal supplemental oxygen on functional respiratory parameters, ventilatory patterns, pulmonary vasculature and quality of life in IPF patients are unknown at present. Furthermore, it is not known whether correction of nocturnal hypoxia prolongs survival. However, in this group of patients, in whom only limited benefits are provided by available treatments, the major crime would be to miss an opportunity. In patients with significant overnight desaturation, there is a sound theoretical basis for suspecting a benefit of supplemental oxygen on quality of life and pulmonary vasculature. There is the outside possibility that treatment with supplemental oxygen could tip the oxidant/antioxidant balance in favour of increased oxidation/oxygen radicals in the fibrotic lungs, with potentially deleterious effects. However, unlike high-dose corticosteroids, it seems highly unlikely that nocturnal supplemental oxygen would lead to significantly increased morbidity and mortality in patients with IPF. Overall, it seems reasonable to use overnight oxygen empirically in patients with significant overnight hypoxia, so as not to risk losing an opportunity. However, large, well-designed studies investigating the prevalence of overnight desaturation (with and without OSA), and assessing the impact of nocturnal supplemental oxygen in patients with IPF and other ILDs, are urgently needed.

\section{Biomarkers in IPF and OSA}

A biological marker or biomarker is a characteristic that is objectively measured and evaluated as an indicator of normal biological processes, pathological processes or biological responses to a therapeutic intervention [72]. The ideal biomarker should 1) be sensitive and specific for the disease, 2) correlate with disease severity, 3) be involved in causal (possibly multiple) pathways, and 4) be measured early in the course of the disease and inexpensively [73]. Plainly, the ideal biomarkers of IPF and OSA still do not exist and this is the challenge for the future.

Unfortunately, until now, there are still no common biomarkers in IPF and OSA and, thus, we will focus on each disease separately. In recent years, there has been a burst of publications regarding the quest for the ideal biomarker in IPF [74], which has been driven mainly by two main issues. Firstly, there is an imperative need for a definite and early diagnosis of IPF, as both have prognostic implications [75]. Moreover, a definite diagnosis in IPF is achievable in only $50 \%$ of cases. In the rest, surgical lung biopsy is warranted but it is not always feasible because of the severe condition of the patient and the presence of comorbidities [76]. Secondly, because the disease has a completely unpredictable course characterised by stability or slow progression over a period of years, rapid deterioration or even periods of relative stability punctuated by events of rapid decline, the knowledge of who is going to progress or not is important for the stratification of the disease. Recently, there was an attempt to combine biomarkers with demographic (age and sex) and functional (FVC) characteristics [77, 78], as it was observed that this combination provides better outcome prediction.

We have passed into a new era of the therapy of IPF, with two IPF-specific drugs [7, 8, 79, 80], and a third issue has emerged in the quest for the ideal biomarker for IPF. Future studies need to focus on the search for biomarkers with the property of identifying who is going to respond or not to these drugs.

To date, a number of potential markers of IPF have been identified and can be divided in three groups: diagnostic, disease susceptibility and prognostic (table 2).

The quest for biomarkers in OSA is justified by: 1) the need to diagnose the disease accurately as frequently signs and symptoms may be under-recognised or not recognised at all by non-experts, and consequently, PSG, the most important diagnostic tool, is not used; 2) the need to follow-up patients and evaluate their adherence to treatment; and finally, 3) the need to find adequate end-points for future clinical trials. A number of biomarkers suggested for OSA is shown in table $3[99,100]$.

The use of these biomarkers has been justified by: 1) the enhancement of inflammation by sleep deprivation, hypoxaemia and excess adipose tissue in obese patients; 2) the activation of the sympathetic nervous system due to apnoea and oxygen desaturation, which leads to the development of arterial hypertension; 3) the association between OSA and diabetes mellitus; and 4) the production of reactive oxygen species due to episodes of hypoxaemia and subsequent desaturation.

As stated earlier, there have been very few studies on biomarkers in patients with both diseases. Recently, it was observed that in patients with IPF and sleep disordered breathing, CCL18, a prognostic biomarker of IPF [90], was associated with increased carbon dioxide during sleep, suggesting the presence of hypoventilation [100].

An issue that should be stressed is that the presence of confounding factors in both disorders that may affect the discovery of the ideal biomarker. Smoking status, age and coexistence of other chronic lung diseases can alter the levels of some biomarkers in IPF, and the same can happen with the presence of obesity, type 2 diabetes, cardiovascular diseases, the metabolic syndrome, insulin resistance and smoking 
TABLE 2 Biomarkers in idiopathic pulmonary fibrosis

Diagnostic biomarkers

Disease susceptibility biomarkers
$\mathrm{KL}-6$ [81]

SP-A and SP-D [81]

YKL-40 [81]

MMP-1 and MMP-7 [81]

CXCL13 [82]

Telomerase genes [83-85]

Telomere length [86]

MUC5B $[87,88]$

TOLLIP-SPPL2C [89]

\section{Prognostic biomarkers}

$\mathrm{KL}-6$ [81]

CCL-18 [90]

YKL-40 [81]

Circulating fibrocytes [91]

CD28 [92]

CD28/ICOS/Lck/ITK [77]

MMP-7/ICAM-1/IL-8/VCAM-1/S100A12 [78]

MUC5B [93]

Cilium-associated genes [94]

Antibodies against periplakin [95]

Antibodies against HSP-70 [96]

LOXL2 [97]

CXCL13 [82]

TLR-3 [98]

SP: surfactant protein; MMP: matrix metalloproteinase; MUC: mucin; TOLLIP: Toll-interacting protein; SPPL: signal peptide peptidase-like; CD: cluster of differentiation; ICOS: inducible T-cell costimulatory; Lck: lymphocyte-specific protein tyrosine kinase; ITK: interleukin-2-inducible T-cell kinase; ICAM: intercellular adhesion molecule; IL: interleukin; VCAM: vascular cell adhesion molecule; HSP: heat shock protein; LOXL: lysyl oxidase-like; TLR: Toll-like receptor.

in OSA [101]. Thus, these conditions must be taken into account when evaluating the strength of a potential biomarker.

Until now, most of the studies regarding biomarkers in IPF have been hampered by a number of limitations such as small size of retrospective cohorts, inclusion of mixed populations, lack of independent validation and absence of longitudinal sampling. However, recent studies have taken advantage of multicentric studies with bigger cohorts and modern validation methods.

\section{Epigenetic and microRNA signatures in IPF and OSA}

Apart from the major control of gene expression at the level of initiation of transcription, which is exerted by transcription factors, human cells exploit additional mechanisms, such as epigenetic modifications and microRNA (miRNA) expression, to achieve restricted expression at a variety of spatial and temporal levels $[102,103]$. The epigenetic information is conveyed through a large number of chemical modifications, including DNA methylation, reversible histone modifications and ATP-dependent nucleosomal remodelling [104]. Chromatin modifications are multiple, functionally linked and cross-regulated while they are dynamically modulated in the course of various biological processes [105-107], generating a

\section{TABLE 3 Biomarkers in obstructive sleep apnoea}

Inflammatory markers (CRP, IL-6, TNF- $\alpha$, fibrinogen, CCL-18)

Systemic hypertension

Carotid intimal-medial thickness

Metabolic markers (HbA1c)

Markers of oxidative stress (8-isoprostane, IL-6, nitric oxide)

Others (cysteine)

CRP: C-reactive protein; IL: interleukin; TNF: tumour necrosis factor; HbA1c: glycated haemoglobin. 
complex code that is superimposed on the genetic code [108]. Factors such as the environment, diet or ageing may influence these modifications [109].

Recent experimental evidence has emerged that DNA methylation is an important mechanism involved in IPF [110-112]. A comparative analysis of global DNA methylation examining 27578 CpG islands combined with gene expression patterns from IPF and normal samples revealed that both DNA methylation and expression profiles were altered in IPF [113]. The mechanistic role of DNA methylation in IPF was further supported by the inverse correlation between the expression levels of 16 IPF-associated genes and their corresponding DNA methylation status. Moreover, the expression levels of DNA methyltransferase (Dnmt)-3a and Dnmt-3b were increased in IPF samples, further supporting the role of DNA methylation in the disease. A different study highlighted an additional epigenetic signature in IPF samples, as they presented an intermediate DNA methylation profile, compared with normal and lung cancer samples [114].

Surprisingly, the hypothesis that epigenetic changes might be associated with OSA has not been investigated in adults with OSA. Intriguingly though, the same hypothesis has been tested in two recent studies in paediatric OSA exploring the association between epigenetic modifications and divergent inflammatory phenotypes. The first study reported that the FOXP3 gene, which regulates expression of T-regulatory lymphocytes, exhibited a higher probability for high methylation levels in children with OSA who manifest an inflammatory phenotype. The prevalence of FOXP3 DNA hypermethylation was dose-dependently increased in relation to the severity of OSA, as defined by the AHI. Moreover, FOXP3 DNA methylation levels not only correlated with inflammatory biomarkers and serum lipids, such as high-sensitivity C-reactive protein, myeloid-related protein-8/14 and apolipoprotein B, but were also associated with AHI and BMI z-scores [115]. The hypothesis of epigenetic modifications in children with OSA was further supported by KHEIRANDISH-GozAL et al. [116], who showed that a promoter region of the eNOS gene displayed significantly higher methylation levels in the OSA-abnormal group than the OSA-normal or control groups. This hypermethylation pattern was nicely coupled with significantly reduced eNOS mRNA expression levels in the OSA-abnormal group in comparison with the OSA-normal group, further supporting the association between DNA methylation and the OSA phenotype.

In terms of histone modifications, there is no information available on either IPF or OSA so far, mainly due to technical limitations in the number of cells and the chromatin immunoprecipitation experiments required.

Further studies characterising epigenomic profiles, particularly employing next-generation sequencing-based methods, should be carried out, not only associating epigenetic alterations with clinical parameters of the disease but also identifying epigenetic signatures serving as potential molecular biomarkers. Moreover, the very promising studies in OSA and childhood should be expanded in adults with this particular sleep disorder.

A substantially greater amount of information has been obtained over the past few years regarding the role of miRNAs in the pathogenesis of IPF [110, 117-119]. miRNAs comprise a set of noncoding RNAs, regulating the expression of specific genes at the post-transcriptional level and determining fundamental properties of the cells [120]. The first report revealing the potential role of miRNAs in IPF demonstrated impressively by array analysis that $10 \%$ of the miRNAs were significantly different between IPF and control samples [121]. A series of elegant studies followed evaluating the miRNAs expression levels in IPF, such as the downregulation of members of Let-7, miR-29, miR-30, miR-31, miR-200, miR-326 and the miR-17-92 cluster, and the upregulation of miR-155 and miR-21 [121-127].

More intriguingly, the effects of the altered miRNA expression levels detected in IPF specimens were clearly correlated to key features of the disease, such as the epithelial-mesenchymal transition in lung epithelial cells or the regulation of transforming growth factor- $\beta 1$, Wnt, sonic hedgehog, p53 and vascular endothelial growth factor pathways, and complex regulatory networks. Both DNA methylation and miRNA mechanisms are able to crosstalk, as a feedback loop is formed between Dnmt-1 and the miR-17-92 cluster resulting in the upregulation of hallmark genes in IPF [125].

Further research on the role of epigenetic markers will hopefully create exciting possibilities not only for the understanding of IPF pathogenesis in depth but also the improvement in clinical diagnosis and treatment of patients with IPF. The detection of specific miRNA alterations in serum samples of IPF patients suggests that miRNA could be used as a noninvasive screening tool for IPF in routine clinical practice, establishing more reliable biomarkers [126, 128]. Novel treatment strategies based on epigenetic drugs, such as 5-aza-2'-deoxycytidine and decitabine [129], or exogenous administration of miRNAs, could also prove beneficial for management of IPF patients. Finally, studies on specific miRNAs in relation to OSA would bring into light potential pathogenetic mechanisms of OSA, identify individuals with increased susceptibility to OSA and provide useful information for the development of novel therapeutic strategies. 


\section{Conclusion}

It is now believed that IPF and sleep disorders can coexist in the same patient, possibly sharing some common pathogenetic pathways. With regards to IPF, the current recommendation is immediate referral to a specialised centre upon suspicion for definite diagnosis of the disease itself as well as of the associated comorbidities. Management of comorbidities by specialists is equally important because if left untreated, they can be the cause of deterioration of the underlying IPF. Moreover, referral to specialists can lead to the inclusion of patients in clinical trials and research studies, such as on epigenetic changes and biomarkers, which can provide useful insights into the pathogenesis and, consequently, the management of both IPF and sleep disorders.

\section{References}

1 Raghu G, Collard HR, Egan JJ, et al. An official ATS/ERS/JRS/ALAT statement: idiopathic pulmonary fibrosis: evidence-based guidelines for diagnosis and management. Am J Respir Crit Care Med 2011; 183: 788-824.

2 Margaritopoulos GA, Romagnoli M, Poletti V, et al. Recent advances in the pathogenesis and clinical evaluation of pulmonary fibrosis. Eur Respir Rev 2012; 21: 48-56.

3 McNicholas WT, Verbraecken J, Marin JM. Sleep disorders in COPD: the forgotten dimension. Eur Respir Rev 2013; 22: 365-375.

$4 \quad$ McNicholas WT. Impact of sleep in COPD. Chest 2000; 117: Suppl. 2, 48S-53S.

5 Chaouat A, Weitzenblum E, Krieger J, et al. Association of chronic obstructive pulmonary disease and sleep apnea syndrome. Am J Respir Crit Care Med 1995; 151: 82-86.

6 Machado MC, Vollmer WM, Togeiro SM, et al. CPAP and survival in moderate-to-severe obstructive sleep apnoea syndrome and hypoxaemic COPD. Eur Respir J 2010; 35: 132-137.

$7 \quad$ King TE Jr, Bradford WZ, Castro-Bernardini S, et al. A phase 3 trial of pirfenidone in patients with idiopathic pulmonary fibrosis. N Engl J Med 2014; 370: 2083-2092.

8 Richeldi L, du Bois RM, Raghu G, et al. Efficacy and safety of nintedanib in idiopathic pulmonary fibrosis. N Engl J Med 2014; 370: 2071-2082.

9 Mermigkis C, Bouloukaki I, Schiza SE. Obstructive sleep apnea in patients with interstitial lung diseases: past and future. Sleep Breath 2013; 17: 1127-1128.

10 Rasche K, Orth MJ. Sleep and breathing in idiopathic pulmonary fibrosis. Physiol Pharmacol 2009; 60: Suppl. 5, 13-14.

11 Agarwal S, Richardson B, Krishnan V, et al. Interstitial lung disease and sleep: what is known? Sleep Med 2009; 10: 947-951.

12 American Thoracic Society/European Respiratory Society international multidisciplinary consensus classification of the idiopathic interstitial pneumonias. Am J Respir Crit Care Med 2002; 165: 277-304.

13 American Academy of Sleep Medicine. The AASM Manual for the scoring of sleep and associated events. Rules, Terminology and Technical Specifications. Westchester, AASM, 2014.

14 Bye $\mathrm{P}$, Issa $\mathrm{F}$, Berthon-Jones $\mathrm{M}$, et al. Studies of oxygenation during sleep in patients with interstitial lung disease. Am Rev Respir Dis 1984; 129: 27-32.

15 Perez-Padilla R, West P, Lertzman M, et al. Breathing during sleep in patients with interstitial lung disease. Am Rev Respir Dis 1985; 132: 224-229.

16 Mermigkis C, Stagaki E, Amfilochiou A, et al. Sleep quality and associated daytime consequences in patients with idiopathic pulmonary fibrosis. Med Princ Pract 2009; 18: 10-15.

17 McNicholas W, Coffey M, Fitzgerald M. Ventilation and gas exchange during sleep in patients with interstitial lung disease. Thorax 1986; 41: 777-782.

18 Mermigkis C, Chapman J, Golish J, et al. Sleep-related breathing disorders in patients with idiopathic pulmonary fibrosis. Lung 2007; 185: 173-178.

19 Aronson RM, Carley DW, Onal E. Upper airway muscle activity and the thoracic volume dependence of upper airway resistance. J Appl Physiol 1991; 70: 430-438.

20 Sériès $\mathrm{F}$, Cormier $\mathrm{Y}$, Lampron $\mathrm{N}$, et al. Increasing the functional residual capacity may reverse obstructive sleep apnea. Sleep 1988; 11: 349-353.

21 Sériès F, Cormier Y, Lampron N, et al. Influence of lung volume in sleep apnea. Thorax 1989; 44: 52-57.

22 Heinzer RC, Stanchina ML, Malhotra A, et al. Lung volume and continuous positive airway pressure requirements in obstructive sleep apnea. Am J Respir Crit Care Med 2005; 172: 114-117.

23 Lancaster LH, Mason WR, Parnell JA, et al. Obstructive sleep apnea is common in idiopathic pulmonary fibrosis. Chest 2009; 136: 772-778.

24 Mermigkis C, Stagaki E, Tryfon S, et al. How common is sleep-disordered breathing in patients with idiopathic pulmonary fibrosis? Sleep Breath 2010; 14: 387-390.

25 Pihtili A, Bingol Z, Kiyan E, et al. Obstructive sleep apnea is common in patients with interstitial lung disease. Sleep Breath 2013; 17: 1281-1288.

26 Young T, Shahar E, Nieto FJ, et al. Predictors of sleep-disordered breathing in community-dwelling adults: the Sleep Heart Health Study. Arch Intern Med 2002; 162: 893-900.

27 Lederer DJ, Jelic S, Bhattacharya J, et al. Is obstructive sleep apnea a cause of idiopathic pulmonary fibrosis? Arch Pathol Lab Med 2012; 136: 470.

28 Leslie KO. Idiopathic pulmonary fibrosis may be a disease of recurrent, tractional injury to the periphery of the aging lung: a unifying hypothesis regarding etiology and pathogenesis. Arch Pathol Lab Med 2012; 136: 591-600.

29 Peppard PE, Young T, Palta M, et al. Prospective study of the association between sleep-disordered breathing and hypertension. N Engl J Med 2000; 342: 1378-1384.

30 Shahar E, Whitney CW, Redline S, et al. Sleep-disordered breathing and cardiovascular disease: cross-sectional results of the Sleep Heart Health Study. Am J Respir Crit Care Med 2001; 163: 19-25.

31 Mermigkis C, Mermigkis D, Varouchakis G, et al. CPAP treatment in patients with idiopathic pulmonary fibrosis and obstructive sleep apnea-therapeutic difficulties and dilemmas. Sleep Breath 2012; 16: 1-3. 

and obstructive sleep apnea: does it offer a better quality of life and sleep? Sleep Breath 2013; 17: 1137-1143.

33 Kolilekas L, Manali E, Vlami KA, et al. Sleep oxygen desaturation predicts survival in idiopathic pulmonary fibrosis. J Clin Sleep Med 2013; 9: 593-601.

34 Mermigkis C, Bouloukaki I, Antoniou K, et al. Obstructive sleep apnea should be treated in patients with idiopathic pulmonary fibrosis. Sleep Breath 2015; 19: 385-391.

35 Campen MJ, Shimoda LA, O’Donnell CP. Acute and chronic cardiovascular effects of intermittent hypoxia in C57BL/6J mice. J Appl Physiol 2005; 99: 2028-2035.

36 Fagan KA. Pulmonary hypertension in mice intermittent hypoxia. J Appl Physiol 2001; 90: $2502-2507$.

37 McGuire M, Bradford A. Chronic intermittent hypercapnic hypoxia increases pulmonary arterial pressure and haematocrit in rats. Eur Respir J 2001; 18: 279-285.

38 Wang Z, Li AY, Guo QH, et al. Effects of cyclic intermittent hypoxia on ET-1 responsiveness and endothelial dysfunction of pulmonary arteries in rats. PLoS One 2013; 8: e58078.

39 Sajkov D, McEvoy RD. Obstructive sleep apnea and pulmonary hypertension. Prog Cardiovasc Dis 2009; 51: 363-370.

40 Minai OA, Ricaurte B, Kaw R, et al. Frequency and impact of pulmonary hypertension in patients with obstructive sleep apnea syndrome. Am J Cardiol 2009; 104: 1300-1306.

41 Arias MA, Rio FG, Fernandez AA, et al. Pulmonary hypertension in obstructive sleep apnoea: effects of continuous positive airway pressure. Eur Heart J 2006; 27: 1106-1113.

42 Sajkov D, Wang T, Saunders NA, et al. Continuous positive airway pressure treatment improves pulmonary hemodynamics in patients with obstructive sleep apnea. Am J Respir Crit Care Med 2002; 165: 152-158.

43 Dumitrascu R, Tiede H, Eckermann J, et al. Sleep apnea in precapillary pulmonary hypertension. Sleep Med 2013; 14: 247-251.

44 Nathan SD, Shlobin OA, Ahmad S, et al. Serial development of pulmonary hypertension in patients with idiopathic pulmonary fibrosis. Respiration 2008; 76: 288-294.

45 Nadrous HF, Pellikka PA, Krowka MJ, et al. Pulmonary hypertension in patients with idiopathic pulmonary fibrosis. Chest 2005; 128: 2393-2399.

46 Lettieri CJ, Nathan SD, Barnett SD, et al. Prevalence and outcomes of pulmonary arterial hypertension in advanced idiopathic pulmonary fibrosis. Chest 2006; 129: 746-752.

47 Corte TJ, Wort SJ, Gatzoulis MA, et al. Pulmonary vascular resistance predicts early mortality in patients with diffuse fibrotic lung disease and suspected pulmonary hypertension. Thorax 2009; 64: 883-888.

48 Modrykamien AM, Gudavalli R, McCarthy K, et al. Echocardiography, 6-minute walk distance, and distance-saturation product as predictors of pulmonary arterial hypertension in idiopathic pulmonary fibrosis. Respir Care 2010; 55: 584-588.

49 Harari S. Out of proportion pulmonary hypertension: a paradigm for rare diseases. Chest 2012; 142: 1087-1088.

50 Clark M, Cooper B, Singh S, et al. A survey of nocturnal hypoxaemia and health related quality of life in patients with cryptogenic fibrosing alveolitis. Thorax 2001; 56: 482-486.

51 Corte TJ, Wort SJ, Talbot S, et al. Elevated nocturnal desaturation index predicts mortality in interstitial lung disease. Sarcoidosis Vasc Diffuse Lung Dis 2012; 29: 41-50.

52 Hira HS, Sharma RK. Study of oxygen saturation, breathing pattern and arrhythmias in patients of interstitial lung disease during sleep. Ind J Chest Dis Allied Sci 1997; 39: 157-162

53 Pitsiou G, Bagalas V, Boutou A, et al. Should we routinely screen patients with idiopathic pulmonary fibrosis for nocturnal hypoxemia? Sleep Breath 2013; 17: 447-448.

54 Turner GA, Lower EE, Corser BC, et al. Sleep apnea in sarcoidosis. Sarcoidosis Vasc Diffuse Lung Dis 1997; 14: 61-64.

55 Aydogdu M, Ciftci B, Firat GS, et al. Interstisyel akciger hastalarinda polisomnografi ile uyku ozelliklerinin degerlendirilmesi [Assessment of sleep with polysomnography in patients with interstitial lung disease]. Tuberk Toraks 2006; 54: 213-221.

56 Hamada K, Nagai S, Tanaka S, et al. Significance of pulmonary arterial pressure and diffusion capacity of the lung as prognosticator in patients with idiopathic pulmonary fibrosis. Chest 2007; 131: 650-656.

57 Corte TJ, Wort SJ, Gatzoulis MA, et al. Elevated brain natriuretic peptide predicts mortality in interstitial lung disease. Eur Respir J 2010; 36: 819-825.

58 Corte TJ, Keir GJ, Dimopoulos K, et al. Bosentan in pulmonary hypertension associated with fibrotic idiopathic interstitial pneumonia. Am J Respir Crit Care Med 2014; 190: 208-217.

59 Hudgel DW, Martin RJ, Capehart M, et al. Contribution of hypoventilation to sleep oxygen desaturation in chronic obstructive pulmonary disease. J Appl Physiol 1983; 55: 669-677.

60 Fletcher EC, Gray BA, Levin DC. Nonapneic mechanisms of arterial oxygen desaturation during rapid-eye-movement sleep. J Appl Physiol 1983; 54: 632-639.

61 Coccagna G, Lugaresi E. Arterial blood gases and pulmonary and systemic arterial pressure during sleep in chronic obstructive pulmonary disease. Sleep 1978; 1: 117-124.

62 Boysen PG, Block AJ, Wynne JW, et al. Nocturnal pulmonary hypertension in patients with chronic obstructive pulmonary disease. Chest 1979; 76: 536-542.

63 Weitzenblum E, Muzet A, Ehrhart M, et al. Variations nocturnes des gaz du sang et de la pression artérielle pulmonaire chez les bronchitiques chroniques insuffisants respiratoires [Nocturnal changes in blood gases and pulmonary arterial pressure in chronic bronchitis patients with respiratory insufficiency]. Nouv Presse Med 1982; 11: $1119-1122$

64 Fletcher EC, Levin DC. Cardiopulmonary hemodynamics during sleep in subjects with chronic obstructive pulmonary disease. The effect of short- and long-term oxygen. Chest 1984; 85: 6-14.

65 Raeside DA, Brown A, Patel KR, et al. Ambulatory pulmonary artery pressure monitoring during sleep and exercise in normal individuals and patients with COPD. Thorax 2002; 57: 1050-1053.

66 Nocturnal Oxygen Therapy Trial Group. Continuous or nocturnal oxygen therapy in hypoxemic chronic obstructive lung disease: a clinical trial. Ann Intern Med 1980; 93: 391-398.

67 Report of the Medical Research Council Working Party. Long term domiciliary oxygen therapy in chronic hypoxic cor pulmonale complicating chronic bronchitis and emphysema. Lancet 1981; 1: 681-686. 
Weitzenblum E, Sautegeau A, Ehrhart M, et al. Long-term oxygen therapy can reverse the progression of pulmonary hypertension in patients with chronic obstructive pulmonary disease. Am Rev Respir Dis 1985; 131: 493-498.

Zieliński J, Tobiasz M, Hawryłkiewicz I, et al. Effects of long-term oxygen therapy on pulmonary hemodynamics in COPD patients: a 6-year prospective study. Chest 1998; 113: 65-70.

Crockett AJ, Cranston JM, Antic N. Domiciliary oxygen for interstitial lung disease. Cochrane Database Syst Rev 2001; CD002883.

Lubin M, Chen H, Elicker B, et al. A comparison of health-related quality of life in idiopathic pulmonary fibrosis and chronic hypersensitivity pneumonitis. Chest 2014; 145: 1333-1338.

Biomarkers Definitions Working Group. Biomarkers and surrogate endpoints: preferred definitions and conceptual framework. Clin Pharmacol Ther 2001; 69: 89-95.

Shih JL, Malhotra A. Could vitamins be helpful to patients with sleep apnea? Chest 2011; 139: $237-238$.

Antoniou KM, Margaritopoulos GA, Tomassetti S, et al. Interstitial lung disease. Eur Respir Rev 2014; 23 : 40-54. Antoniou KM, Symvoulakis EK, Margaritopoulos GA, et al. Early diagnosis of IPF: time for a primary-care case-finding initiative? Lancet Respir Med 2014; 2: e1.

Travis WD, Costabel U, Hansell DM, et al. An official American Thoracic Society/European Respiratory Society statement: update of the international multidisciplinary classification of the idiopathic interstitial pneumonias. Am J Respir Crit Care Med 2013; 188: 733-748.

Herazo-Maya JD, Noth I, Duncan SR, et al. Peripheral blood mononuclear cell gene expression profiles predict poor outcome in idiopathic pulmonary fibrosis. Sci Transl Med 2013; 5: 205 ral36.

Richards TJ, Kaminski N, Baribaud F, et al. Peripheral blood proteins predict mortality in idiopathic pulmonary fibrosis. Am J Respir Crit Care Med 2012; 185: 67-76.

Antoniou KM, Margaritopoulos GA, Siafakas NM. Pharmacological treatment of idiopathic pulmonary fibrosis: from the past to the future. Eur Respir Rev 2013; 22: 281-291.

Cottin V, Crestani B, Valeyre D, et al. Diagnosis and management of idiopathic pulmonary fibrosis: French practical guidelines. Eur Respir Rev 2014; 23: 193-214.

Vij R, Noth I. Peripheral blood biomarkers in idiopathic pulmonary fibrosis. Transl Res 2012; 159: $218-227$.

Vuga LJ, Tedrow JR, Pandit KV, et al. C-X-C motif chemokine 13 (CXCL13) is a prognostic biomarker of idiopathic pulmonary fibrosis. Am J Respir Crit Care Med 2014; 189: 966-974.

Armanios MY, Chen JJ, Cogan JD, et al. Telomerase mutations in families with idiopathic pulmonary fibrosis. N Engl J Med 2007; 356: 1317-1326.

Tsakiri KD, Cronkhite JT, Kuan PJ, et al. Adult-onset pulmonary fibrosis caused by mutations in telomerase. Proc Natl Acad Sci USA 2007; 104: 7552-7557.

Mushiroda T, Wattanapokayakit S, Takahashi A, et al. A genome-wide association study identifies an association of a common variant in TERT with susceptibility to idiopathic pulmonary fibrosis. J Med Genet 2008; 45: 654-656.

Alder JK, Chen JJ, Lancaster L, et al. Short telomeres are a risk factor for idiopathic pulmonary fibrosis. Proc Natl Acad Sci USA 2008; 105: 13051-13056.

Seibold MA, Wise AL, Speer MC, et al. A common MUC5B promoter polymorphism and pulmonary fibrosis. N Engl J Med 2011; 364: 1503-1512.

Zhang Y, Noth I, Garcia JG, et al. A variant in the promoter of MUC5B and idiopathic pulmonary fibrosis. N Engl J Med 2011; 364: 1576-1577.

Noth I, Zhang Y, Ma S-F, et al. Genetic variants associated with idiopathic pulmonary fibrosis susceptibility and mortality: a genome-wide association study. Lancet Respir Med 2013; 1: 309-317.

Prasse A, Probst C, Bargagli E, et al. Serum CC-chemokine ligand 18 concentration predicts outcome in idiopathic pulmonary fibrosis. Am J Respir Crit Care Med 2009; 179: 717-723.

Moeller A, Gilpin SE, Ask K, et al. Circulating fibrocytes are an indicator of poor prognosis in idiopathic pulmonary fibrosis. Am J Respir Crit Care Med 2009; 179: 588-594.

Gilani SR, Vuga LJ, Lindell KO, et al. CD28 down-regulation on circulating CD4 T-cells is associated with poor prognoses of patients with idiopathic pulmonary fibrosis. PLoS One 2010; 5: e8959.

Peljto AL, Zhang Y, Fingerlin TE, et al. Association between the MUC5B promoter polymorphism and survival in patients with idiopathic pulmonary fibrosis. JAMA 2013; 309: 2232-2239.

Yang IV, Coldren CD, Leach SM, et al. Expression of cilium-associated genes defines novel molecular subtypes of idiopathic pulmonary fibrosis. Thorax 2013; 68: 1114-1121.

Taillé C, Grootenboer-Mignot S, Boursier C, et al. Identification of periplakin as a new target for autoreactivity in idiopathic pulmonary fibrosis. Am J Respir Crit Care Med 2011; 183: 759-766.

Kahloon RA, Xue J, Bhargava A, et al. Patients with idiopathic pulmonary fibrosis with antibodies to heat shock protein 70 have poor prognoses. Am J Respir Crit Care Med 2013; 187: 768-775.

Chien JW, Richards TJ, Gibson KF, et al. Serum lysyl oxidase-like 2 levels and idiopathic pulmonary fibrosis disease progression. Eur Respir J 2014; 43: 1430-1438.

O’Dwyer DN, Armstrong ME, Trujillo G, et al. The Toll-like receptor 3 L412F polymorphism and disease progression in idiopathic pulmonary fibrosis. Am J Respir Crit Care Med 2013; 188: 1442-1450.

Montesi SB, Bajwa EK, Malhotra A. Biomarkers of sleep apnea. Chest 2012; 142: 239-245.

Lee RN, Kelly E, Nolan G, et al. Disordered breathing during sleep and exercise in idiopathic pulmonary fibrosis and the role of biomarkers. QJM 2015; 108: 315-323.

Arnardottir ES, Mackiewicz M, Gislason T, et al. Molecular signatures of obstructive sleep apnea in adults: a review and perspective. Sleep 2009; 32: 447-470.

Sato F, Tsuchiya S, Meltzer SJ, et al. MicroRNAs and epigenetics. FEBS J 2011; 278: 1598-1609.

Saetrom P, Snove O Jr, Rossi JJ. Epigenetics and microRNAs. Pediatr Res 2007; 61: 17R-23R.

Bernstein BE, Meissner A, Lander ES. The mammalian epigenome. Cell 2007; 128: 669-681.

Bhaumik SR, Smith E, Shilatifard A. Covalent modifications of histones during development and disease pathogenesis. Nat Struct Mol Biol 2007; 14: 1008-1016.

Fischle W. Talk is cheap - cross-talk in establishment, maintenance, and readout of chromatin modifications. Genes Dev 2008; 22: 3375-3382. 
Kouzarides T. Chromatin modifications and their function. Cell 2007; 128: 693-705. Kampranis SC, Tsichlis PN. Histone demethylases and cancer. Adv Cancer Res 2009; 102: 103-169.

Yang IV, Schwartz DA. Epigenetic control of gene expression in the lung. Am J Respir Crit Care Med 2011; 183: 1295-1301.

Yang IV. Epigenomics of idiopathic pulmonary fibrosis. Epigenomics 2012; 4: 195-203.

Yang IV, Schwartz DA. Epigenetics of idiopathic pulmonary fibrosis. Transl Res 2015; 165: 48-60.

Sun X, He Y, Huang C, et al. The epigenetic feedback loop between DNA methylation and microRNAs in fibrotic disease with an emphasis on DNA methyltransferases. Cell Signal 2013; 25: 1870-1876.

Sanders YY, Ambalavanan N, Halloran B, et al. Altered DNA methylation profile in idiopathic pulmonary fibrosis. Am J Respir Crit Care Med 2012; 186: 525-535.

PLoS One 2012; 7: e33770.

Kim J, Bhattacharjee R, Khalyfa A, et al. DNA methylation in inflammatory genes among children with obstructive sleep apnea. Am J Respir Crit Care Med 2012; 185: 330-338.

Kheirandish-Gozal L, Khalyfa A, Gozal D, et al. Endothelial dysfunction in children with obstructive sleep apnea is associated with epigenetic changes in the eNOS gene. Chest 2013; 143: 971-977.

Santangelo S, Scarlata S, Zito A, et al. Genetic background of idiopathic pulmonary fibrosis. Expert Rev Mol Diagn 2013; 13: 389-406.

Vancheri C. Common pathways in idiopathic pulmonary fibrosis and cancer. Eur Respir Rev 2013; 22: 265-272. Pandit KV, Milosevic J, Kaminski N. MicroRNAs in idiopathic pulmonary fibrosis. Transl Res 2011; 157: 191-199.

Nakahara K, Carthew RW. Expanding roles for miRNAs and siRNAs in cell regulation. Curr Opin Cell Biol 2004; 16: 127-133.

Respir Crit Care Med 2010; 182: 220-229.

Liu G, Friggeri A, Yang Y, et al. miR-21 mediates fibrogenic activation of pulmonary fibroblasts and lung fibrosis. J Exp Med 2010; 207: 1589-1597.

Yang S, Banerjee S, de Freitas A, et al. Participation of miR-200 in pulmonary fibrosis. Am J Pathol 2012; 180: 484-493.

4 Yang S, Xie N, Cui H, et al. miR-31 is a negative regulator of fibrogenesis and pulmonary fibrosis. FASEB J 2012; 26: 3790-3799.

Dakhlallah D, Batte K, Wang Y, et al. Epigenetic regulation of miR-17 92 contributes to the pathogenesis of pulmonary fibrosis. Am J Respir Crit Care Med 2013; 187: 397-405.

Li P, Zhao GQ, Chen TF, et al. Serum miR-21 and miR-155 expression in idiopathic pulmonary fibrosis. J Asthma 2013; 50: 960-964.

Das S, Kumar M, Negi V, et al. MicroRNA-326 regulates profibrotic functions of transforming growth factor-beta in pulmonary fibrosis. Am J Respir Cell Mol Biol 2014; 50: 882-892.

Li P, Li J, Chen T, et al. Expression analysis of serum microRNAs in idiopathic pulmonary fibrosis. Int J Mol Med 2014; 33: 1554-1562.

Rosas IO, Yang IV. The promise of epigenetic therapies in treatment of idiopathic pulmonary fibrosis. Am J Respir Crit Care Med 2013; 187: 336-338. 